TRANSACTIONS OF THE

AMERICAN MATHEMATICAL SOCIETY

Volume 358, Number 1, Pages 359-371

S 0002-9947(05)03676-7

Article electronically published on March 31, 2005

\title{
UNRAMIFIED COHOMOLOGY OF CLASSIFYING VARIETIES FOR EXCEPTIONAL SIMPLY CONNECTED GROUPS
}

\author{
SKIP GARIBALDI
}

\begin{abstract}
Let $B G$ be a classifying variety for an exceptional simple simply connected algebraic group $G$. We compute the degree 3 unramified Galois cohomology of $B G$ with values in $(\mathbb{Q} / \mathbb{Z})^{\prime}(2)$ over an arbitrary field $F$. Combined with a paper by Merkurjev, this completes the computation of these cohomology groups for $G$ semisimple simply connected over all fields.

These computations provide another family of examples of simple simply connected groups $G$ such that $B G$ is not stably rational.
\end{abstract}

Let $G$ be an algebraic group over a field $F$ with an embedding $\rho: G \hookrightarrow S L_{n}$ over $F$. We call the quotient variety $S L_{n} / G$ a classifying space of $G$ and denote it by $B G$. The goal of this paper is to compute the unramified cohomology of $B G$, defined as follows. Write $H^{d}(F)$ for the Galois cohomology group $H^{d}\left(\operatorname{Gal}(F),(\mathbb{Q} / \mathbb{Z})^{\prime}(d-1)\right)$, where $(\mathbb{Q} / \mathbb{Z})^{\prime}(d-1)=\underline{\lim }_{n} \boldsymbol{\mu}_{n}^{\otimes(d-1)}$ for $n$ not divisible by the characteristic of $F$. For each $d \geq 2$, define ${\overrightarrow{H_{\mathrm{nr}}^{d}}}^{n}(B G / F)$ (or simply $H_{\mathrm{nr}}^{d}(B G)$ ) to be the intersection of the kernels of the residue homomorphisms

$$
\partial_{v}: H^{d}(F(B G)) \rightarrow H^{d-1}(F(v))
$$

as $v$ ranges over the discrete valuations of $F(B G)$ over $F$. The natural homomorphism $H^{d}(F) \rightarrow H_{\mathrm{nr}}^{d}(B G / F)$ is split by evaluation at the distinguished point of $B G$; this gives a direct sum decomposition of $H_{\mathrm{nr}}^{d}(B G)$, and we denote the complement of $H^{d}(F)$ by $H_{\mathrm{nr}}^{d}(B G)_{\text {norm }}$. This group depends only on $G$ and $F$, and not on $\rho$ or $n$ [M02, 2.3].

This paper completes the computation of $H_{\mathrm{nr}}^{3}(B G / F)_{\text {norm }}$ for $G$ semisimple simply connected and $F$ arbitrary. The computation is quickly reduced to the case where $G$ is simple simply connected [M02, §4]. In [M02], $H_{\mathrm{nr}}^{3}(B G)_{\text {norm }}$ was computed for $G$ simple and classical. We compute it for the remaining cases, where $G$ is exceptional, that is, where $G$ is of type $G_{2},{ }^{3} D_{4},{ }^{6} D_{4}, F_{4}, E_{6}, E_{7}$, or $E_{8}$.

Main Theorem 0.2. Let $G$ be a simple simply connected exceptional algebraic group defined over a field $F$. Then

$$
H_{\mathrm{nr}}^{3}(B G)_{\mathrm{norm}}= \begin{cases}\mathbb{Z} / 2 & \begin{array}{l}
\text { if char } F \neq 2, G \text { is of type }{ }^{3} D_{4}, \text { and } G \text { has } \\
\text { a nontrivial Tits algebra, }
\end{array} \\
0 & \text { otherwise. }\end{cases}
$$

(See 5.3 for an explanation of the characteristic $\neq 2$ hypothesis.)

The general motivation for studying $H_{\mathrm{nr}}^{d}(X)$ is that it can sometimes detect if $X$ is not stably rational; see [C, pp. 35-39]. It was an open question whether $B G$ is

Received by the editors August 15, 2003 and, in revised form, March 21, 2004.

2000 Mathematics Subject Classification. Primary 11E76; Secondary 17B25, 20 G10. 
stably rational for $G$ semisimple simply connected. The first counterexamples were provided in [M02], where Merkurjev exhibited groups $G$ of type ${ }^{2} A_{n},{ }^{2} D_{3}$, and ${ }^{1} D_{4}$ with $H_{\mathrm{nr}}^{3}(B G)_{\text {norm }} \neq 0$, hence with $B G$ not stably rational. The results here give another class of such $G$ 's; see Corollary 7.2 .

Our basic tool is that one can compute $H_{\mathrm{nr}}^{3}(B G)_{\text {norm }}$ by inspecting the ramification of the Rost invariant of $G$; see $\mathrm{M} 02$ or Lemma 5.2. Many questions are settled by hopping along the chain of inclusions

$$
G_{2} \subset D_{4} \subset F_{4} \subset E_{6} \subset E_{7} \subset E_{8}
$$

of split groups; see $\S \S 6$ and 5.5

The most interesting part of the proof is where we show that the mod 4 portion of the Rost invariant is ramified for groups of type ${ }^{2} E_{6}$. We prove (in Theorem 3.1) that every isotropic trialitarian group embeds in a group of type ${ }^{2} E_{6}$ with trivial Tits algebras. This settles the question, since the mod 4 portion of the Rost invariant for groups of type ${ }^{6} D_{4}$ is easily shown to be ramified (Lemma 6.3). The proof of Theorem 3.1 uses Galois descent and interpretations of exceptional groups as acting on nonassociative algebras.

Remark 0.4. Computations of $H_{\mathrm{nr}}^{d}(X / F)$ in the literature for $X$ a smooth variety (e.g., a classifying variety) typically assume that $F$ is algebraically closed. The examples of nonrational classifying varieties $B G$ provided here and in 1 M02 require that $F$ is not algebraically closed (e.g., $F=\mathbb{Q}$ ).

\section{Vocabulary}

An (affine) algebraic group is simple if it is $\neq 1$, is connected, and has no noncentral connected normal subgroups over an algebraic closure. (These groups are often called "absolutely almost simple".) Simple groups are classified in, e.g., [KMRT, Ch. VI]. We say that a group is of type $T_{n}$ if it is simple with root system of type $T_{n}$ and of type ${ }^{t} T_{n}$ if additionally the absolute Galois group of $F$ acts as a group of automorphisms of order $t$ on the Dynkin diagram.

Let $V$ be a finite-dimensional irreducible representation of an algebraic group $G$ over $F$. The $F$-algebra $\operatorname{End}_{G}(V)$ is a skew field by Schur's Lemma, and it is finite dimensional over $F$; it is called a Tits algebra for $G$. If it is a (commutative) field, we say that it is trivial.

The Dynkin index $n_{G}$ of a simple simply connected algebraic group $G$ is a natural number which depends only upon the type of $G$ and the (Schur) indices of its Tits algebras. The value of $n_{G}$ can be found in [M03, App. B], for example:

\begin{tabular}{||c||c|c|c|c|c|c|c|c||}
\hline type of $G$ & $G_{2}$ & $\begin{array}{c}{ }^{3,6} D_{4} \text {, all Tits } \\
\text { alg's trivial }\end{array}$ & $\begin{array}{c}3,6 \\
{ }^{3} D_{4} \text {, some Tits } \\
\text { alg's nontrivial }\end{array}$ & $F_{4}$ & ${ }^{1} E_{6}$ & ${ }^{2} E_{6}$ & $E_{7}$ & $E_{8}$ \\
\hline$n_{G}$ & 2 & 6 & 12 & 6 & 6 & 12 & 12 & 60 \\
\hline
\end{tabular}

We have functors $H^{1}(*, G)$ and $H^{3}(*)$ which take a field extension of $F$ and give a pointed set and abelian group, respectively. A degree 3 invariant of $G$ with values in $(\mathbb{Q} / \mathbb{Z})^{\prime}(2)$ is a morphism of functors

$$
H^{1}(*, G) \longrightarrow H^{3}(*)
$$

which takes base points to base points. Such invariants are often called "normalized". We write $\operatorname{Inv}^{3}(G)$ for the abelian group of such invariants. (Clearly, this definition makes sense for every algebraic group $G$ over $F$.) 
Let $p=\operatorname{char} F$ if the characteristic is prime and $p=1$ otherwise. Write $n_{G}=$ $p^{k} n_{G}^{\prime}$, where $n_{G}^{\prime}$ is a natural number prime to $p$. The group $\operatorname{Inv}^{3}(G)$ is cyclic of order $n_{G}^{\prime}$. It has a canonical generator $r_{G}$ which we call the Rost invariant of $G$. (This is the prime-to- $p$ part of what is called the Rost invariant in M03. I do not know how to define a residue map for the $p$-primary part.)

For $\alpha: H \rightarrow G$ a map between simple simply connected algebraic groups over $F$, there is a positive integer $n_{\alpha}$ called the Rost multiplier or "Dynkin index" of $\alpha$; see [M03, §7]. It has the properties: $n_{H}$ divides $n_{\alpha} n_{G}$ and for every extension $E$ of $F$, the composition

$$
H^{1}(E, H) \stackrel{\alpha}{\longrightarrow} H^{1}(E, G) \stackrel{r_{G}}{\longrightarrow} H^{3}(E)
$$

is $n_{\alpha} r_{H}$.

The standard inclusions in (0.3) all have Rost multiplier 1. This observation goes back to Dyn, p. 192]. Excepting $G_{2}$, this can be seen as follows: The inclusion of $D_{4}$ into $E_{8}$ arises from the natural inclusion of root systems. It is easy to check from the definition that the Rost multiplier is 1 . Since multipliers are nonnegative integers and the Rost multiplier of a composition is the product of the Rost multipliers M03, 7.9], the intervening Rost multipliers are all 1. An alternative, explicit argument for the inclusion $F_{4} \subset E_{6}$ can be found in [Ga01, 2.4].

All inclusions of groups of type $G_{2}, D_{4}, F_{4}, E_{n}$ occurring in this paper are conjugates of the standard inclusions of split groups over an algebraic closure of the base field. Since Rost multipliers are unchanged by field extensions [M03, 7.9.4], the multipliers for these inclusions are all 1.

$$
\text { 2. } A_{2} \subset D_{4}
$$

2.1. In this section, we assume that $F$ contains a primitive cube root of unity and hence has characteristic $\neq 3$. Let $L$ be a cubic Galois extension of $F$; by Kummer theory it is obtained by adjoining a cube root of some element $\lambda \in F^{*}$. We write $(\lambda)$ for the corresponding class in $F^{*} / F^{* 3}=H^{1}\left(F, \boldsymbol{\mu}_{3}\right)$, where $\boldsymbol{\mu}_{3}$ is the algebraic group of cube roots of unity.

The short exact sequence $1 \rightarrow \boldsymbol{\mu}_{3} \rightarrow S L_{3} \rightarrow P G L_{3} \rightarrow 1$ induces a connecting homomorphism $\delta: H^{1}\left(F, P G L_{3}\right) \rightarrow H^{2}\left(F, \boldsymbol{\mu}_{3}\right)$.

Lemma 2.2. Continue the hypotheses of $\$ 2.1$. Let $G$ be the quasi-split simply connected group of type ${ }^{3} D_{4}$ associated with the extension $L / F$. Then $G$ contains a subgroup isomorphic to $P G L_{3}$ such that for every extension $E$ of $F$ the diagram

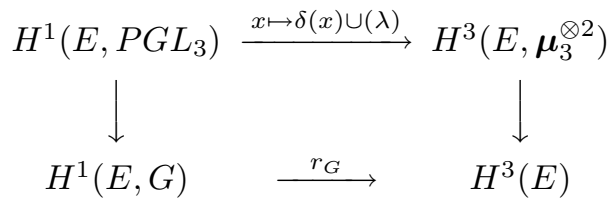

commutes up to sign, where the arrow on the right comes from the natural map $\boldsymbol{\mu}_{3}^{\otimes 2} \rightarrow(\mathbb{Q} / \mathbb{Z})^{\prime}(2)$.

Proof. We have maps

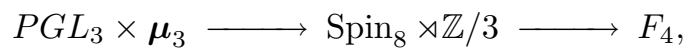

where $F_{4}$ denotes the split algebraic group of that type and we identify $\boldsymbol{\mu}_{3}$ with $\mathbb{Z} / 3$ using the primitive cube root of unity in $F$. The first map comes from the fact that $P G L_{3}=\operatorname{Aut}\left(M_{3}(F)\right)$ preserves the subspace of trace zero elements in $M_{3}(F)$; see 
KMRT, pp. 504, 505]. The second map comes from the Springer decomposition of Albert algebras; see [KMRT, 38.7]. The group $\mathbb{Z} / 3$ acts on $\operatorname{Spin}_{8}$ in a manner which cyclically permutes the vector and half-spin representations and fixes $P G L_{3}$ elementwise. The map $\operatorname{Spin}_{8} \rightarrow F_{4}$ has Rost multiplier 1 .

When we twist the groups in (2.3) by $(\lambda)$ and restrict to connected components, we obtain the sequence

$$
P G L_{3} \stackrel{\iota}{\longrightarrow} G \stackrel{\sigma}{\longrightarrow} F_{4} .
$$

The map $H^{1}(\sigma \iota)$ sends a class $[A] \in H^{1}\left(E, P G L_{3}\right)$ of a central simple $E$-algebra of degree 3 to the class of the first Tits construction $[J(A, \lambda)] \in H^{1}\left(F, F_{4}\right)$; see [KMRT, 39.9]. The composition $r_{F_{4}} \circ H^{1}(\sigma \iota)$ is, up to sign, the composition of $\delta$ with the cup product $\cup \cup(\lambda)$ by [KMRT, p. 537]. Since $\sigma$ has Rost multiplier 1, $r_{F_{4}} \circ H^{1}(\sigma)=r_{G}$, and the lemma is proved.

$$
\text { 3. }{ }^{3} D_{4},{ }^{6} D_{4} \subset{ }^{2} E_{6}
$$

In this section, we assume that $F$ has characteristic $\neq 2$. A simple algebraic group is said to be trialitarian if it is of type ${ }^{3} D_{4}$ or ${ }^{6} D_{4}$. It is well known that every quasi-split simply connected trialitarian group is a subgroup of the split $F_{4}$, hence of every simply connected quasi-split $E_{6}$. In this section, we prove:

Theorem 3.1 (char $F \neq 2$ ). Let $T$ be a trialitarian simply connected group over $F$ which is $F$-isotropic but not $F$-quasi-split. Let $K$ be a quadratic extension of $F$ such that $T$ is $K$-quasi-split. Then there exists a simply connected group $G$ of type ${ }^{2} E_{6}$ over $F$ such that

(1) all of $G$ 's Tits algebras are trivial;

(2) $G$ is of type ${ }^{1} E_{6}$ over $K$; and

(3) $T$ is a subgroup of $G$ with Rost multiplier 1.

Given a $T$ as in the first sentence of Theorem 3.1 such a $K$ always exists by Ga98, 0.1]. We postpone the proof of the theorem until the end of this section.

3.2. Let $\mathfrak{C}$ be the split Cayley algebra over $F$ with canonical involution (also known as conjugation) $\pi_{\mathfrak{C}}$. Fix the basis $u_{1}, \ldots, u_{8}$ of $\mathfrak{C}$ as in Ga98 and Ga01 so that the bilinear norm form $\mathfrak{n}(x, y)=x \pi_{\mathfrak{C}}(y)+y \pi_{\mathfrak{C}}(x)$ is given by $\mathfrak{n}\left(u_{i}, u_{j}\right)=\delta_{(i+j), 9}$ (Kronecker delta). Write $\sigma$ for the involution on $G L(\mathfrak{C})$ which is adjoint for $\mathfrak{n}$.

Let $R$ denote the subgroup of $G L(\mathfrak{C})^{\times 3}$ consisting of so-called related triples of proper similitudes of $\mathfrak{n}$; see [Ga98, §1] or [KMRT, §35] for a definition. This group is reductive with center of rank 2; its derived subgroup consists of triples $\underline{t}=\left(t_{0}, t_{1}, t_{2}\right)$ with $t_{i} \in S O(\mathfrak{n})$ for all $i$ and is isomorphic to $\operatorname{Spin}_{8}$ [KMRT, 35.7].

The group $S_{3}=\left\langle r, \pi \mid r^{3}=\pi^{2}=1, \pi r=r^{2} \pi\right\rangle$ acts on $R$ via

$$
{ }^{r} \underline{t}=\left(t_{1}, t_{2}, t_{0}\right) \quad \text { and } \quad{ }^{\pi}\left(t_{0}, t_{1}, t_{2}\right)=\left(\pi_{\mathfrak{C}} t_{0} \pi_{\mathfrak{C}}, \pi_{\mathfrak{C}} t_{2} \pi_{\mathfrak{C}}, \pi_{\mathfrak{C}} t_{1} \pi_{\mathfrak{C}}\right) .
$$

Define $R \rtimes S_{3}$ to be the Cartesian product $R \times S_{3}$ with multiplication

$$
(\underline{t}, \alpha) \cdot\left(\underline{t}^{\prime}, \beta\right)=\left(\underline{t} \cdot \underline{t}^{\prime}, \alpha \beta\right) .
$$

The split Albert algebra $J$ has as an underlying vector space the matrices in $M_{3}(\mathfrak{C})$ fixed by the conjugate transpose. With that in mind, we may write a general element of $J$ as in (3.4) below where $\varepsilon_{i} \in F, c_{i} \in \mathfrak{C}$, and the entries given as - are forced by symmetry. The algebra $J$ has a canonically determined norm form; write $\operatorname{Inv}(J)$ for the group of norm isometries. 
There is an injection $g: R \rtimes S_{3} \hookrightarrow \operatorname{Inv}(J)$ defined by

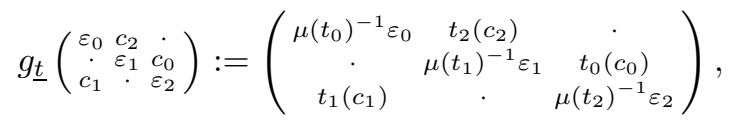

$$
\begin{aligned}
& g_{r}\left(\begin{array}{ccc}
\varepsilon_{0} & c_{2} & \dot{c}_{0} \\
\dot{c} & \varepsilon_{1} & c_{0} \\
c_{1} & \cdot & \varepsilon_{2}
\end{array}\right)=\left(\begin{array}{ccc}
\varepsilon_{1} & c_{0} & \cdot \\
\cdot & \varepsilon_{2} & c_{1} \\
c_{2} & \cdot & \varepsilon_{0}
\end{array}\right) \text {, and } g_{\pi}\left(\begin{array}{ccc}
\varepsilon_{0} & c_{2} & \dot{\varepsilon}_{0} \\
\dot{c_{1}} & \varepsilon_{1} & c_{0} \\
c_{1} & \varepsilon_{2}
\end{array}\right)=\left(\begin{array}{ccc}
\varepsilon_{0} & \pi_{\mathfrak{C}} c_{1} & \cdot \\
\cdot & \varepsilon_{2} & \pi_{\mathfrak{C}} c_{0} \\
\pi_{\mathfrak{C}} c_{2} & \cdot & \varepsilon_{1}
\end{array}\right) \text {. }
\end{aligned}
$$

Here $\mu\left(t_{i}\right)$ denotes the factor by which $t_{i}$ scales the norm $\mathfrak{n}$. That is, $\mathfrak{n}\left(t_{i} x, t_{i} y\right)=$ $\mu\left(t_{i}\right) \mathfrak{n}(x, y)$ for all $x, y \in \mathfrak{C}$.

3.3. Construction of a quasi-split ${ }^{2} E_{6}$. The algebra $J$ is also endowed with a nondegenerate symmetric bilinear form $s$ defined by

$$
s(x, y)=\operatorname{Tr}_{J}(x y)=\sum_{i=0}^{2}\left[\varepsilon_{i} \nu_{i}+\mathfrak{n}\left(c_{i}, d_{i}\right)\right]
$$

for

$$
x=\left(\begin{array}{ccc}
\varepsilon_{0} & c_{2} & \dot{c} \\
\dot{c_{1}} & \varepsilon_{1} & c_{0} \\
c_{1} & \cdot & \varepsilon_{2}
\end{array}\right), \quad y=\left(\begin{array}{ccc}
\nu_{0} & d_{2} & \dot{c}^{\prime} \\
\dot{d_{1}} & \nu_{1} & d_{0} \\
\dot{d}_{1} & \cdot & \nu_{2}
\end{array}\right) .
$$

For each $f \in G L(J)$, there is a unique $f^{\dagger} \in G L(J)$ such that $s\left(f(x), f^{\dagger}(y)\right)=s(x, y)$ for all $x, y \in J$.

The map $f \mapsto f^{\dagger}$ restricts to automorphisms of $\operatorname{Inv}(J)$ and $R \rtimes S_{3}$ defined over $F$. We have

$$
r^{\dagger}=r, \quad \pi^{\dagger}=\pi, \quad \text { and } \quad \underline{t}^{\dagger}=\left(\sigma\left(t_{0}\right)^{-1}, \sigma\left(t_{1}\right)^{-1}, \sigma\left(t_{2}\right)^{-1}\right) .
$$

Let $\iota$ be a generator for $\operatorname{Gal}(K / F)$. We define the groups $E_{6}^{K}$ and $H$ to be the groups $\operatorname{Inv}(J)$ and $R$ with twisted $\iota$-actions. For $f$ a $K$-point, we set ${ }^{\iota} f=\iota f^{\dagger} \iota$ where the action on the left is the new action and juxtaposition denotes the usual action. The group $E_{6}^{K}$ is quasi-split of type ${ }^{2} E_{6}$.

We consider $E_{6}^{K}$ as a closed subgroup of $G L(V)$ as follows. Let $V$ be the $F$ subspace of $(J \otimes K) \oplus(J \otimes K)$ fixed by the $\iota$-semilinear automorphism

$$
\left(j \otimes k, j^{\prime} \otimes k^{\prime}\right) \mapsto\left(j^{\prime} \otimes \iota\left(k^{\prime}\right), j \otimes \iota(k)\right) .
$$

The homomorphism

$$
\operatorname{Inv}(J) \rightarrow G L(J \oplus J) \quad \text { given by } f \mapsto f \oplus f^{\dagger}
$$

defines an injective $F$-homomorphism $E_{6}^{K} \rightarrow G L(V)$. It is the usual 54-dimensional representation of $E_{6}^{K}$; it is irreducible over $F$.

3.5. Proof of Theorem 3.1. Since $T$ is isotropic and not quasi-split, it has a Tits algebra which is a nonsplit quaternion algebra $Q$ over a cubic extension $L$ of $F$ Ga98, 0.1]. Put $K=F(\sqrt{b})$. Since $T$ is $K$-quasi-split, $Q$ is split by $L(\sqrt{b})$, hence $Q$ is of the form $(a, b)_{L}$ for some $a \in L^{*}$ such that $N_{L / F}(a)=1$ [KMRT, 43.9]. Since $Q$ is not split over $L$, it is not split over the normal closure $L^{c}$ of $L / F$ Ga98, 3.2]. In particular, $L^{c}$ does not contain a square root of $b$, so $P=K \otimes_{F} L^{c}$ is a quadratic field extension of $L^{c}$.

To simplify our argument, we assume that $L$ is not Galois over $F$, so $\operatorname{Gal}\left(L^{c} / F\right)$ is isomorphic to $S_{3}$. (This is the case that will be used in the rest of the paper. The other case - where $L$ is Galois over $F$ - is only easier.) Then, the group $\operatorname{Gal}(P / F)$ is isomorphic to $S_{3} \times \boldsymbol{\mu}_{2} \cong \mathbb{Z} / 6 \rtimes \boldsymbol{\mu}_{2}$, where the factor of $\mathbb{Z} / 6$ corresponds to the subgroup $\operatorname{Gal}(P / \Delta)$ for $\Delta$ the unique quadratic extension of $F$ in $L^{c}$. We fix 
generators $\zeta:=\left(r^{-1},-1\right) \in S_{3} \times \boldsymbol{\mu}_{2}$ (which generates the copy of $\left.\mathbb{Z} / 6\right)$ and $\tau=(\pi, 1)$ (which generates the copy of $\boldsymbol{\mu}_{2}$ in $\mathbb{Z} / 6 \rtimes \boldsymbol{\mu}_{2}$ corresponding to $\operatorname{Gal}(P / L(\sqrt{b})$ )).

We construct the group $G$ by descent as follows. We identify $E_{6}^{K}$ - and hence $H \rtimes S_{3}$ - with a closed subgroup of $G L(V)$ as in $₫ 3.3$. We call an additive homomorphism $f: V \otimes P \rightarrow V \otimes P \varpi$-semilinear if there is some $\varpi \in \operatorname{Gal}(P / F)$ such that

$$
f(p v)=\varpi(p) f(v) \quad \text { for all } p \in P \text { and } v \in V \otimes P .
$$

Let $\widetilde{G L}(V)$ denote the (abstract) group of such maps $f$. We define a group homomorphism $\phi: \operatorname{Gal}(P / F) \rightarrow \widetilde{G L}(V)$ such that $\phi(\varpi)$ is $\varpi$-semilinear for all $\varpi$ and $T(F)$ and $G(F)$ are the subgroups of $\operatorname{Spin}_{8}(P)$ and $E_{6}^{K}(P)$ commuting with $\phi(\varpi)$ for all $\varpi$.

Define $\underline{t}=\left(t_{0}, t_{1}, t_{2}\right) \in G L(\mathfrak{C})^{\times 3}$ by setting $t_{i}=m_{i} P$ for

$$
m_{i}=\operatorname{diag}\left(1, \rho^{i}(a),-\rho^{i}(a), \rho^{i+2}(a)^{-1}, \rho^{i+1}(a)^{-1},-1,1, \rho^{i}(a)\right)
$$

with $\rho:=\zeta^{2}$, and $P$ the matrix permuting the basis vectors as $(12)(36)(45)(78)$, for the basis of $\mathfrak{C}$ fixed in $\$ 3.2$ above. Since $N_{L / F}(a)=1, \underline{t}$ is a related triple by Ga98, 1.5(3), 1.6, 1.8]. Set

$$
\phi(\zeta)=\underline{t} r \zeta \quad \text { and } \quad \phi(\tau)=\pi \tau .
$$

We have

$$
\zeta \underline{t} \zeta^{-1}=\left(\sigma\left(\zeta\left(t_{0}\right)\right), \sigma\left(\zeta\left(t_{1}\right)\right), \sigma\left(\zeta\left(t_{2}\right)\right)\right)^{-1}=\left(\sigma\left(t_{2}\right), \sigma\left(t_{0}\right), \sigma\left(t_{1}\right)\right)^{-1}=r^{-1} \underline{t}^{-1} r,
$$

since $\sigma\left(t_{i}\right)=t_{i}$ for all $i$. Hence

$$
\begin{gathered}
\phi(\zeta)^{2}=r^{2} \zeta^{2}, \quad \phi(\zeta)^{3}=\underline{t} \zeta^{3}, \quad \text { and } \\
\phi(\zeta)^{6}=\operatorname{Id}_{V \otimes P} .
\end{gathered}
$$

Since $\pi$ and $\tau$ commute, we have

$$
\phi(\tau)^{2}=\operatorname{Id}_{V \otimes P} .
$$

Since

$$
\phi(\zeta)^{5}=\phi(\zeta)^{2} \cdot \phi(\zeta)^{3}=\underline{t} r^{2} \zeta^{5},
$$

and $\pi \tau$ and $\underline{t}$ commute, it is easy to verify that

$$
\phi(\tau) \phi(\zeta)=\phi(\zeta)^{5} \phi(\tau) .
$$

Equations (3.7)-(3.9) give that (3.6) defines a homorphism $\phi: \operatorname{Gal}(P / F) \rightarrow$ $\widetilde{G L}(V)$. Then the set map $z: \operatorname{Gal}(P / F) \rightarrow H \rtimes S_{3}$ defined by $z_{\varpi}:=\phi(\varpi) \varpi^{-1}$ is in fact a 1-cocycle. (This correspondence between groups of semilinear transformations and 1-cocycles is well-explained in [Jac, §3].) Set $G$ to be the twisted group $\left(E_{6}^{K}\right)_{z}$; it automatically satisfies (2). Since $z$ takes values in the simply connected group $E_{6}^{K},(1)$ holds.

Since the values of $z$ normalize the subgroup $\operatorname{Spin}_{8}$ of $E_{6}^{K}$, the twisted group $\left(\operatorname{Spin}_{8}\right)_{z}$ is a subgroup of $G$. The inclusion has Rost multiplier 1 since the inclusion $\operatorname{Spin}_{8} \subset E_{6}^{K}$ over an algebraic closure arises from the natural inclusion of root systems $D_{4} \subset E_{6}$. The restriction of $z$ to $\operatorname{Spin}_{8}$ is the descent given in Ga98, 4.7] to construct $T$, i.e., $\left(\operatorname{Spin}_{8}\right)_{z}$ is isomorphic to $T$, hence (3).

Remark 3.10. The isotropic group $G$ occurring in Theorem 3.1 is typically not quasi-split, even over $L$. This can be seen by examining the mod 2 portion of the Rost invariant for $(z) \in H^{1}\left(P / L, E_{6}^{K}\right)$, which is typically nontrivial by [Ga01, 6.7]. 


\section{A construction}

The purpose of this section is to construct a suitable extension of $F$ over which we may apply Theorem 3.1 .

Proposition 4.1. Let $F$ be a field of characteristic $\neq 2$, and let $K$ be a quadratic extension of $F$. There is a regular extension $E$ of $F$ and a group $T$ of type ${ }^{6} D_{4}$ over $E$ such that $T$ is E-isotropic and $\left(E \otimes_{F} K\right)$-quasi-split, but not E-quasi-split.

Presumably one could prove Proposition 4.1 by applying M96 to produce a group $T_{0}$ of type ${ }^{6} D_{4}$ whose Tits algebras have index 8 and then extending scalars to function fields of transfers of generalized Severi-Brauer varieties so that the Tits algebras of $T_{0}$ have index 2 and are split by a quadratic extension of the base field. Then by Ga98 there is a group $T$ as in Proposition 4.1 with the same Tits algebras as in $T_{0}$. We give a low-tech argument here.

Lemma 4.2 (char $F \neq 2)$. For $p, q \in F^{*}$, the ring $L=F(t)[x] /\left(x^{3}+p x+q t\right)$ is a separable cubic field extension of $F(t)$ which is regular over $F$ and not Galois over $F(t)$. There is a prolongation of the $t$-adic valuation on $F(t)$ to $L$ which is unramified with residue degree 1 and with respect to which $x$ has value 1.

Proof. If $L$ is not a field, then there is some $a \in F(t)$ such that $a^{3}+p a+q t=0$. Since $a$ is integral over the unique factorization domain $F[t]$, it belongs to $F[t]$, so it makes sense to speak of the degree of $a$. In particular, at least two of the terms $a^{3}$, $p a$, and $q t$ must have the same degree, which is also the maximum of the degrees. This implies that $a$ cannot have positive degree. But then $q t$, with degree 1 , is the unique term of maximal degree, which is a contradiction.

Since $p, q$ are in $F^{*}$, the discriminant $-4 p^{3}-27 q^{2} t^{2}$ of $L$ is not 0 , hence $L$ is separable over $F$. An argument similar to the one in the preceding paragraph shows that the discriminant is not a square in $F(t)$ : Any square root $b \in F(t)$ of the discriminant would belong to $F[t]$ and have degree 1 . Then the coefficient of $t$ in $b^{2}$ would be nonzero. Thus $L$ is not Galois over $F(t)$. If $\ell \in L \backslash F(t)$ is algebraic over $F$, then $L$ is generated by $\ell$ as an $F(t)$-algebra and the discriminant of the extension $L / F(t)$ comes from $F^{*} / F^{* 2}$, which is a contradiction. Hence $F$ is algebraically closed in $L$ and $L$ is regular over $F$.

Hensel's Lemma gives that $x^{3}+p x+t q$ has a linear factor of the form $x-\pi$ in $F((t))[x]$, where $\pi$ has $t$-adic value 1 . The map $x \mapsto \pi$ gives an isomorphism of $L$ with the subfield $F(t)(\pi)$ of $F((t))$, and the $t$-adic valuation obviously extends to $L$ so that $x$ has value 1 . Since $F((t))$ is the completion of $F(t)$ with respect to the $t$-adic valuation and hence is unramified with residue degree 1 , the claims about ramification and residue degree of our prolongation to $L$ follow.

Lemma 4.3 (char $F \neq 2$ ). Let $p, b \in F^{*}$ be such that the quaternion algebra $(p, b)_{F}$ is nonsplit. Let $L$ be as in Lemma 4.2. Then the quaternion algebra $(x, b)_{L}$ is nonsplit and is not isomorphic to $(-q t, b)_{L}$.

Proof. Since $N_{L / F(t)}(x)=-q t$, the corestriction of $(x, b)_{L}$ down to $F(t)$ is Brauerequivalent to $(-q t, b)_{F(t)}$. This algebra is split if and only if the quadratic form $\langle 1,-b, q t\rangle$ is isotropic over $F(t)$. Over the completion $F((t))$, this form has residue forms $\langle 1,-b\rangle$ and $\langle q\rangle$. Since the algebra $(p, b)_{F}$ is nonsplit, the first form is anisotropic, hence $\langle 1,-b, q t\rangle$ is anisotropic over $F((t))$ by Springer's Theorem. Thus $(-q t, b)_{F(t)}$ is nonsplit, and hence so is $(x, b)_{L}$. 
For the sake of contradiction, suppose that $(x, b)_{L}$ is isomorphic to $(-q t, b)_{L}$, i.e., the algebra $(-x q t, b)_{L}$ is split. Since

$$
-x(q t)=-x\left(-x^{3}-p x\right)=x^{4}+p x^{2} \equiv x^{2}+p \quad \bmod L^{* 2},
$$

the algebra $\left(x^{2}+p, b\right)_{L}$ is split.

Let $\widehat{L}$ be a completion of $L$ with respect to the prolongation of the $t$-adic valuation on $F(t)$ given by Lemma 4.2. The norm of $\left(x^{2}+p, b\right)_{L}$ is the form $\left\langle 1,-\left(x^{2}+p\right),-b, b\left(x^{2}+p\right)\right\rangle$ over $L$. Since $x$ has value 1 , over $\widehat{L}$ this form has one residue form $\langle 1,-p,-b, b p\rangle$ over the residue field $F$. This is the norm of the algebra $(p, b)_{F}$, which is anisotropic because the algebra is nonsplit. By Springer's Theorem, the norm of $\left(x^{2}+p, b\right)_{L}$ is anisotropic over $\widehat{L}$, hence the algebra is not $L$-split, which contradicts our assumption that $(x, b)_{L}$ is isomorphic to $(-q t, b)_{L}$.

4.4. Proof of Proposition 4.1. Write $K$ as $F(\sqrt{b})$. Let $F_{0}:=F(p, t)$ for $p, t$ indeterminates. Set $L_{0}:=F_{0}[x] /\left(x^{3}+p x+t\right)$ as in Lemma 4.2 . Set $E$ to be the function field of the Severi-Brauer variety of the quaternion algebra $(-t, b)_{F_{0}}$. Since $E$ is regular over $F_{0}, L:=L_{0} \otimes_{F_{0}} E$ is a field which is cubic and not Galois over $E$; it is the function field of the Severi-Brauer variety of $(-t, b)_{L_{0}}$.

Since $b$ is not a square in $F$, the quaternion algebra $(p, b)_{F_{0}}$ is not split. By Lemma 4.3. $(x, b)_{L_{0}}$ is nonsplit and is not isomorphic to $(-t, b)_{L_{0}}$, hence $(x, b)_{L} \cong$ $(x, b)_{L_{0}} \otimes_{L_{0}} L$ is not split by a well-known theorem of Amitsur. The corestriction $\operatorname{cor}_{L / E}(x, b)_{L}$ is $(-t, b)_{E}$, which is split. Thus there is a simply connected $E$ isotropic - but not $E$-quasi-split — trialitarian group $T$ over $E$ with nontrivial Tits algebra $(x, b)_{L}$ Ga98, 4.7]. It is of type ${ }^{6} D_{4}$ since $L$ is not Galois over $E$. It is $E(\sqrt{b})$-quasi-split since $(x, b)$ is split over $L(\sqrt{b})$ Ga98, 5.6].

\section{RAMIFICATION}

Let $G$ be an algebraic group over $F$. We say that an invariant $a \in \operatorname{Inv}^{3}(G)$ is unramified if the composition

$$
H^{1}(E((t)), G) \stackrel{a}{\longrightarrow} H^{3}(E((t))) \stackrel{\partial}{\longrightarrow} H^{2}(E)
$$

is 0 for every field extension $E$ of $F$. Otherwise we say that $a$ is ramified. The following example is typical:

Example 5.1. Let $F$ be a field with a primitive cube root of unity, and let $L=F\left(\lambda^{1 / 3}\right)$ be a cubic Galois extension of $F$. We claim that the invariant $a \in \operatorname{Inv}^{3}\left(P G L_{3}\right)$ from $\$ 2$ given by $a(x)=\delta(x) \cup(\lambda)$ is ramified.

The set $H^{1}\left(F, P G L_{3}\right)$ classifies degree 3 cyclic central simple algebras $(C, d)$ for $C$ a cubic Galois extension of $F$ and $d \in F^{*}$. Let $[C] \in H^{1}(F, \mathbb{Z} / 3)$ denote the class corresponding to $C$. Then $\delta(C, d) \cup(\lambda)$ is $\pm[C] \cup(d) \cup(\lambda)$ in $H^{3}\left(F, \boldsymbol{\mu}_{3}^{\otimes 2}\right)$. Taking $E=F(u)$ for $u$ an indeterminate and $C=E((t))\left(t^{1 / 3}\right)$ a cubic Galois extension of $E((t))$, we have

$$
\partial[\delta(C, u) \cup(\lambda)]= \pm(u) \cup(\lambda) \quad \in H^{2}(E) .
$$

This is nonzero in $H^{2}(E)$ since $u$ is not a norm from the extension $E\left(\lambda^{1 / 3}\right) / E$. Hence $a$ is ramified, as claimed.

For the rest of the section, we assume that $G$ is simple and simply connected. We write $\operatorname{Inv}_{\mathrm{nr}}^{3}(G)$ for the subset of unramified invariants in $\operatorname{Inv}^{3}(G)$. It is a subgroup since $\partial$ is a group homomorphism. 


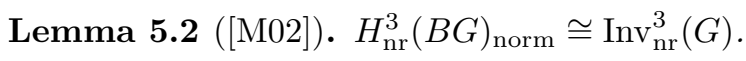

In particular, $H_{\mathrm{nr}}^{3}(B G)_{\text {norm }}$ is necessarily finite; see $\S 1$.

5.3. If $F$ has prime characteristic $p$, then multiplication by $p$ is an isomorphism of $(\mathbb{Q} / \mathbb{Z})^{\prime}(2)$. Hence $H^{3}(F), \operatorname{Inv}^{3}(G)$, and - by Lemma $5.2-H_{\mathrm{nr}}^{3}(B G / F)_{\text {norm }}$ have no nontrivial $p$-torsion.

This explains the hypothesis "char $F \neq 2$ " in the Main Theorem: a simply connected group $G$ of type ${ }^{3} D_{4}$ with nontrivial Tits algebras "should" have $H_{\mathrm{nr}}^{3}(B G)_{\text {norm }}$ equal to $\mathbb{Z} / 2$, but this is impossible in characteristic 2 .

Strongly Inner Lemma 5.4. Let $G$ be a simple simply connected group over $F$, and fix $z \in Z^{1}(F, G)$. The canonical identification $\operatorname{Inv}^{3}(G)=\operatorname{Inv}^{3}\left(G_{z}\right)$ defined by $r_{G} \leftrightarrow r_{G_{z}}$ restricts to an identification $\operatorname{Inv}_{\mathrm{nr}}^{3}(G)=\operatorname{Inv}_{\mathrm{nr}}^{3}\left(G_{z}\right)$.

Groups $G_{z}$ obtained by twisting $G$ by a 1-cocycle $z$ as in the lemma are called strongly inner forms of $G$. Such groups have the same type and Tits algebras as $G$, hence also the same Dynkin index as $G$. In particular, $n_{G}^{\prime}=n_{G_{z}}^{\prime}$.

Proof. Let $E$ be an extension of $F$, and consider the diagram

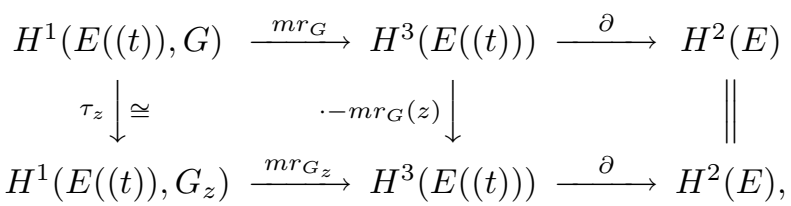

where $\tau_{z}$ is the twisting isomorphism and $m$ is an integer. The left box commutes by [Gi00, p. 76, Lem. 7] or [MPT, 1.7]. The right box commutes because $\partial$ is a group homomorphism and $\partial\left(r_{G}(z)\right)=0$. Hence $m r_{G}$ is ramified if and only if $m r_{G_{z}}$ is.

5.5. Functoriality (homomorphisms). Let $\alpha: H \rightarrow G$ be a morphism of algebraic groups. Then $\alpha$ induces natural homomorphisms

$$
\alpha^{*}: \operatorname{Inv}^{3}(G) \rightarrow \operatorname{Inv}^{3}(H) \quad \text { and } \quad \alpha_{\mathrm{nr}}^{*}: \operatorname{Inv}_{\mathrm{nr}}^{3}(G) \rightarrow \operatorname{Inv}_{\mathrm{nr}}^{3}(H) .
$$

Now suppose that $H$ and $G$ are simple simply connected. If the Rost multiplier of $\alpha$ is 1 , then $n_{H}$ divides $n_{G}$, hence $n_{G}^{\prime} / n_{H}^{\prime}$ divides $n_{G} / n_{H}$. (See $\$ 1$ for definitions.) Also, $\alpha^{*}$ is a surjection with kernel of order $n_{G}^{\prime} / n_{H}^{\prime}$. Then we have: If $\operatorname{Inv}_{\mathrm{nr}}^{3}(H)$ is trivial, then $\operatorname{Inv}_{\mathrm{nr}}^{3}(G)$ is $\left(n_{G} / n_{H}\right)$-torsion.

5.6. Functoriality (scalar extension). Let $K$ be an extension field of $F$, and write $G_{K}$ for $G \times_{F} K$. The restriction homomorphism

$$
\operatorname{res}_{K / F}: \operatorname{Inv}^{3}(G / F) \rightarrow \operatorname{Inv}^{3}(G / K)
$$

is the natural surjection $\mathbb{Z} / n_{G}^{\prime} \rightarrow \mathbb{Z} / n_{G_{K}}^{\prime}$; its kernel is the $\left(n_{G}^{\prime} / n_{G_{K}}^{\prime}\right)$-torsion in $\operatorname{Inv}^{3}(G / F)$. It restricts to a homomorphism

$$
\left(\operatorname{res}_{K / F}\right)_{\mathrm{nr}}: \operatorname{Inv}_{\mathrm{nr}}^{3}(G / F) \rightarrow \operatorname{Inv}_{\mathrm{nr}}^{3}(G / K) .
$$

The kernel of this map is killed by $n_{G}^{\prime} / n_{G_{K}}^{\prime}$, hence by $n_{G} / n_{G_{K}}$. We have: If $\operatorname{Inv}_{\mathrm{nr}}^{3}(G / K)$ is trivial, then $\operatorname{Inv}_{\mathrm{nr}}^{3}(G / F)$ is $\left(n_{G} / n_{G_{K}}\right)$-torsion. 


\section{The CASE Where $G$ HAS TRIVIAL Tits AlgEBRAS}

In this section, we prove:

Proposition 6.1. Let $G$ be a simple simply connected exceptional algebraic group. If $G$ has only trivial Tits algebras, then $\operatorname{Inv}_{\mathrm{nr}}^{3}(G)=0$.

That is, the Main Theorem holds for groups with only trivial Tits algebras by Lemma 5.2. In proving the proposition, we may assume that $G$ is quasi-split by the Strongly Inner Lemma 5.4.

The proposition still holds if the hypothesis "exceptional" is dropped; the classical groups are treated in $\mathrm{M} 02$.

6.2. Type ${ }^{3} D_{4}$. Let $L$ be a separable cubic extension of $F$, such that the quasi-split group $G$ is of type ${ }^{1} D_{4}$ over $L$. Since $G$ is quasi-split, $n_{G_{L}}=2$ and $\operatorname{Inv}_{\mathrm{nr}}^{3}(G / L)=0$ by $[\mathrm{M} 02,8.5]$. Hence $\operatorname{Inv}_{\mathrm{nr}}^{3}(G / F)$ is 3 -torsion by $\$ 5.6$, By $\$ 5.3$ we may assume that char $F \neq 3$.

Let $F^{\prime}$ be the extension obtained from $F$ by adjoining (if not already in $F$ ) a primitive cube root of unity. Then $G$ is still of type ${ }^{3} D_{4}$ over $F^{\prime}$ and the invariants $2 r_{G}$ and $4 r_{G}$ are ramified over $F^{\prime}$ by Lemma 2.2 and Example 5.1. Since $2 r_{G}$ and $4 r_{G}$ are the only nontrivial 3-torsion elements of $\operatorname{Inv}^{3}(G / F)$, we have shown that $\operatorname{Inv}_{\mathrm{nr}}^{3}(G)$ is 0 .

Lemma 6.3. Let $G$ be simple simply connected of type ${ }^{6} D_{4}$ (no restriction on the Tits algebras). Then $\operatorname{Inv}_{\mathrm{nr}}^{3}(G)=0$.

Proof. Let $\Delta$ be the unique quadratic extension of the base field $F$ over which $G$ is of type ${ }^{3} D_{4}$. Let $K$ be a generic quasi-splitting field for $G$ over $\Delta$ as in [KR]. Then $G$ is quasi-split of type ${ }^{3} D_{4}$ over $K$, hence $n_{G_{K}}=6$ and $\operatorname{Inv}_{\mathrm{nr}}^{3}(G / K)=0$ by the ${ }^{3} D_{4}$ case (6.2). We have that $\operatorname{Inv}_{\mathrm{nr}}^{3}(G / F)$ is 2 -torsion by $\$ 5.6$.

Let $L$ be a cubic extension of $F$ over which $G$ is of type ${ }^{2} D_{4}$. Then $n_{G_{L}}$ is 2 or 4 and $\operatorname{Inv}_{\mathrm{nr}}^{3}(G / L)=0$ by $[\mathrm{M} 02,8.5]$. Since $n_{G}=6$ or 12 (as $n_{G_{L}}=2$ or 4 ), $\operatorname{Inv}_{\mathrm{nr}}^{3}(G / F)$ is 3-torsion.

Combining the two previous paragraphs, we find that $\operatorname{Inv}_{\mathrm{nr}}^{3}(G / F)=0$.

6.4. Type $G_{2}$. Here $n_{G}=2$, so we may assume that char $F \neq 2$. The Rost invariant for the split group of type $G_{2}$ is given explicitly in [KMRT, p. 441]. It is the Elman-Lam invariant for 3-Pfister quadratic forms, which is clearly ramified.

6.5. Type $F_{4}$. The split $G_{2}$ is contained in our split group $G$ of type $F_{4}$ with Rost multiplier 1. (See the end of \$1. We omit this sort of observation below.) Since $n_{F_{4}}=6$ and $n_{G_{2}}=2$, the group $\operatorname{Inv}_{\mathrm{nr}}^{3}(G)$ is 3 -torsion by 5.5 and the $G_{2}$ case (@6.4). In characteristic $\neq 3$, the $\bmod 3$ part of the Rost invariant is described in [PR, 3.2], and it is clearly ramified. So $\operatorname{Inv}_{\mathrm{nr}}^{3}(G)=0$.

6.6. Type ${ }^{1} E_{6}$. The split group $G$ of type $E_{6}$ contains a subgroup which is split of type $F_{4}[\mathrm{Sp}, 14.20,14.24]$, and we have $n_{F_{4}}=n_{G}=6$. Hence $\operatorname{Inv}_{\mathrm{nr}}^{3}(G)=0$ by $\$ 5.5$ and the $F_{4}$ case (6.5).

6.7. Type ${ }^{2} E_{6}$. The group $G$ is split by a quadratic extension and $n_{G}=12$. By $\$ 5.6$ and the ${ }^{1} E_{6}$ case $(\$ 6.6), \operatorname{Inv}_{\mathrm{nr}}^{3}(G)$ is 2-torsion, hence we may assume that $\operatorname{char} F \neq 2$.

Let $T$ and $E$ be as in Proposition 4.1. By Theorem 3.1 $T$ is contained in a strongly inner form $G^{\prime}$ of $G$ over $E$. By Lemma 6.3. $\operatorname{Inv}_{\mathrm{nr}}^{3}(T / E)=0$. Since $T$ has 
a nontrivial Tits algebra over $E$, the Dynkin index $n_{T}$ is 12 . Since $n_{G^{\prime}}$ is also 12 , we have $\operatorname{Inv}_{\mathrm{nr}}^{3}\left(G^{\prime} / E\right)=0$. By the Strongly Inner Lemma, $\operatorname{Inv}_{\mathrm{nr}}^{3}(G / E)=0$. Since $G$ is of type ${ }^{2} E_{6}$ over $F$ and $E$, we have $n_{G}=n_{G_{E}}$, hence $\operatorname{Inv}_{\mathrm{nr}}^{3}(G / F)=0$ by 45.6 ,

6.8. Type $E_{7}$. The natural inclusion of root systems gives a split simply connected subgroup of type $E_{6}$ inside the split group $G$ of type $E_{7}$. Since $n_{G}=12$ and $n_{E_{6}}=6$, $\operatorname{Inv}_{\mathrm{nr}}^{3}(G)$ is 2 -torsion by $\$ 5.5$ and the ${ }^{1} E_{6}$ case $(\$ 6.6)$. Hence we may assume that char $F \neq 2$.

Set $F^{\prime}=F(x)$ and $K=F^{\prime}(\sqrt{x})$. There is a quasi-split simply connected group $E_{6}^{K}$ over $F$ of type ${ }^{2} E_{6}$ associated with the extension $K / F^{\prime}$; it injects into $G_{F^{\prime}}$ with Rost multiplier 1 [Ga01, §3]. Since $n_{E_{6}^{K}}$ and $n_{G_{F^{\prime}}}$ are both $12, \operatorname{Inv}_{\mathrm{nr}}^{3}\left(G / F^{\prime}\right)=0$ by $\$ 5.5$ and the ${ }^{2} E_{6}$ case (\$6.7). Since $n_{G}=12$, we have $\operatorname{Inv}_{\mathrm{nr}}^{3}(G / F)=0$.

6.9. Type $E_{8}$. As in the previous cases, we may assume that our group $G$ of type $E_{8}$ is actually split. The natural inclusion of root systems gives an embedding of a split simply connected group of type $E_{7}$ in $G$, so $\operatorname{Inv}_{n r}^{3}(G)$ is 5 -torsion by $\$ 5.5$ and the $E_{7}$ case ( $(6.8)$. In particular, we may assume that char $F \neq 5$ by $\$ 5.3$.

Let $F^{\prime}$ be the extension obtained from $F$ by adjoining two indeterminates and (if necessary) a primitive 5 th root of unity. There is an $F^{\prime}$-central division algebra $D$ of dimension $5^{2}$, namely the symbol algebra determined by the two indeterminates. There are obvious copies of $S L_{5}$ in $G$ arising from inclusions of sets of simple roots, and these inclusions have Rost multiplier 1. Arguing in a manner similar to Gi02, §1], one can twist such an inclusion to find a strongly inner form $G^{\prime}$ of $G$ over $F^{\prime}$ and an injection $S L_{1}(D) \hookrightarrow G^{\prime}$. Now $n_{S L_{1}(D)}=5$ [M03, 11.5] and $\operatorname{Inv}_{\text {nr }}^{3}\left(S L_{1}(D) / F^{\prime}\right)=0$ (as can be seen from the explicit formula for the Rost invariant in [M03, p. 138]), hence $\operatorname{Inv}_{\mathrm{nr}}^{3}\left(G^{\prime} / F^{\prime}\right)$ is 12-torsion by $\$ 5.5$, By the Strongly Inner Lemma, $\operatorname{Inv}_{\mathrm{nr}}^{3}\left(G / F^{\prime}\right)$ is 12-torsion. Since the Dynkin index of $G$ is 60 over $F$ and $F^{\prime}, \operatorname{Inv}_{\mathrm{nr}}^{3}(G / F)$ is 12 -torsion by $\$ 5.6$

Combining the two preceding paragraphs gives that $\operatorname{Inv}_{\mathrm{nr}}^{3}(G)$ is 0 . This completes the proof of Proposition 6.1.

\section{Proof of the Main Theorem}

Let $G$ be as in the Main Theorem 0.2 If $G$ has only trivial Tits algebras (e.g., $G$ is of type $G_{2}, F_{4}$, or $E_{8}$ ), then the Main Theorem holds for $G$ by Proposition 6.1 and Lemma 5.2 .

If $G$ is of type $E_{6}$ or $E_{7}$, then we pick a generic quasi-splitting field $K$ of $G$ over $F$. We have $n_{G_{K}}=n_{G}$ and $\operatorname{Inv}_{\mathrm{nr}}^{3}(G / K)=0$ (by Proposition [6.1), hence $\operatorname{Inv}_{\mathrm{nr}}^{3}(G / F)=0$ by $\$ 5.6$.

If $G$ is of type ${ }^{6} D_{4}$, the Main Theorem holds by Lemma 6.3. The remaining case is where $G$ is of type ${ }^{3} D_{4}$ with nontrivial Tits algebras. We have $n_{G}=12$ and as in the proof of Lemma $6.3 \operatorname{Inv}_{\mathrm{nr}}^{3}(G)$ is 2 -torsion. Hence we may assume that char $F \neq 2$.

The only nontrivial 2-torsion element of $\operatorname{Inv}^{3}(G)$ is $6 r_{G}$, so we will complete the proof of the Main Theorem if we show that $6 r_{G}$ is unramified. That is, if we show that for every extension $E$ of $F$, the composition

$$
H^{1}(E((t)), G) \stackrel{6 r_{G_{E((t))}}}{\longrightarrow} H^{3}(E((t))) \stackrel{\partial}{\longrightarrow} H^{2}(E)
$$

is 0 . 
If $G$ is of type ${ }^{1} D_{4}$ over $E$, then it is of type ${ }^{1} D_{4}$ over $E((t))$ and $n_{G_{E((t))}}$ is 2 or 4 [M03, 15.4]. Hence $6 r_{G_{E((t))}}=2 r_{G_{E((t))}}$ and the composition (7.1) is 0 by [M02, $8.2]$.

Otherwise, $G$ is of type ${ }^{3} D_{4}$ over $E$. That is, if $L$ is a cubic Galois extension of $F$ over which $G$ is of type ${ }^{1} D_{4}$, the tensor product $L^{\prime}:=L \otimes_{F} E$ is a cubic field extension of $E$. We have a diagram

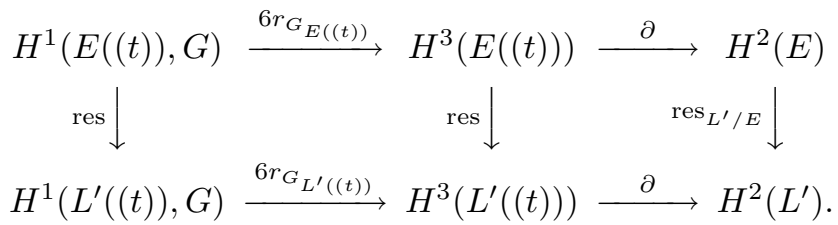

The left box commutes because the Rost invariant is compatible with restriction, and the right box commutes because the extension $E((t)) \subset L^{\prime}((t))$ is unramified, hence the whole diagram commutes. Fix a class $\alpha$ in $H^{1}(E((t)), G)$. Since $G_{L^{\prime}((t))}$ is of type ${ }^{1} D_{4}$, the composition of the two bottom arrows is 0 by the preceding paragraph, and the image of $\alpha$ in $H^{2}\left(L^{\prime}\right)$ is 0 . Let $\beta \in H^{2}(E)$ be the image of $\alpha$; it is 2 -torsion because $6 r_{G}$ is 2 -torsion. Hence

$$
\beta=3 \beta=\operatorname{cor}_{L^{\prime} / E} \operatorname{res}_{L^{\prime} / E}(\beta),
$$

which is 0 by the commutativity of the diagram. This shows that the composition (7.1) - which is the top row of the diagram - is 0 in this case.

Thus $\operatorname{Inv}_{\text {nr }}^{3}(G)=\mathbb{Z} / 2$ for $G$ of type ${ }^{3} D_{4}$ with nontrivial Tits algebras when char $F \neq 2$. This completes the proof of the Main Theorem 0.2 .

Corollary 7.2. Let $G$ be a simply connected group of type ${ }^{3} D_{4}$ or ${ }^{6} D_{4}$ over a field $F$ of characteristic $\neq 2$. If $G$ has a nontrivial Tits algebra, then $B G$ is not stably rational.

Proof. If $G$ is of type ${ }^{3} D_{4}$, this is a direct consequence of the Main Theorem.

If $G$ is of type ${ }^{6} D_{4}$, let $K$ be the unique quadratic extension of $F$ over which $G$ is of type ${ }^{3} D_{4}$. It follows from Ga98, 3.2] that $G$ has nontrivial Tits algebras when $G$ is considered as a $K$-group. Therefore $B G$ is not stably rational as a $K$-variety, hence not as an $F$-variety.

\section{ACKNOWLEDGEMENTS}

Thanks to Adrian Wadsworth for his comments on 84 and to Alexander Merkurjev for suggesting the problem and for enlightening conversations.

\section{REFERENCES}

[C] J.-L. Colliot-Thélène, Birational invariants, purity and the Gersten conjecture, in Ktheory and algebraic geometry: connections with quadratic forms and division algebras (Santa Barbara, CA, 1992) (B. Jacob and A. Rosenberg, eds.), Proceedings of Symposia in Pure Mathematics 58.1 (1995), 1-64. MR1327280(96c:14016)

[Dyn] E.B. Dynkin, Semisimple subalgebras of semisimple Lie algebras, Amer. Math. Soc. Transl. (2) 6 (1957), 111-244 [Russian original: Mat. Sbornik N.S. 30(72) (1952), 349462]. MR0047629(13:904c)

[Ga98] R.S. Garibaldi, Isotropic trialitarian algebraic groups, J. Algebra 210 (1998), 385-418 [DOI 10.1006/jabr.1998.7584]. MR.1662339(99k:20092)

[Ga01] , The Rost invariant has trivial kernel for quasi-split groups of low rank, Comment. Math. Helv. 76 (2001), no. 4, 684-711. MR:1881703 (2003g:20079) 
[Gi00] Ph. Gille, Invariants cohomologiques de Rost en caractéristique positive, K-Theory 21 (2000), 57-100. MR1802626 (2001k:11064)

[Gi02] Algèbres simples centrales de degré 5 et $E_{8}$, Canad. Math. Bull. 45 (2002), no. 3, 388-398. MR.1937674(2004b:16017)

[Jac] N. Jacobson, Triality and Lie algebras of type $D_{4}$, Rend. Circ. Mat. Palermo (2) 13 (1964), 129-153 (= Coll. Math. Papers 71). MR0181705 (31\#5932)

[KMRT] M.-A. Knus, A.S. Merkurjev, M. Rost, and J.-P. Tignol, The book of involutions, Colloquium Publications 44, American Mathematical Society, 1998. MR 1632779 (2000a:16031)

[KR] I. Kersten and U. Rehmann, Generic splitting of reductive groups, Tôhoku Math. J. (2) 46 (1994), 35-70. MR1256727 (95a:20048)

[M96] A.S. Merkurjev, Maximal indexes of Tits algebras, Doc. Math. 1 (1996), 229-243. MR 1405670 (97g:20052)

[M02] Unramified cohomology of classifying varieties for classical simply connected groups, Ann. Sci. École Norm. Sup. (4) 35 (2002), 445-476. MR 1914006 (2003d:11059)

[M03] Rost invariants of simply connected algebraic groups, with a section by S. Garibaldi, in Cohomological invariants in Galois cohomology, University Lecture Series 28, American Mathematical Society, 2003. MR1999385

[MPT] A.S. Merkurjev, R. Parimala, and J.-P. Tignol, Invariants of quasi-trivial tori and the Rost invariant, St. Petersburg Math. J. 14 (2003), 791-821. MR:1970336 (2004c:11045)

[PR] H.P. Petersson and M.L. Racine, An elementary approach to the Serre-Rost invariant of Albert algebras, Indag. Math. (N.S.) 7 (1996), no. 3, 343-365. MR1621373 (99j:17045)

[Sp] T.A. Springer, Jordan algebras and algebraic groups, Ergebnisse der Mathematik und ihrer Grenzgebiete, vol. 75, Springer-Verlag, 1973. MR0379618(52:523)

Department of Mathematics \& Computer Science, Emory University, Atlanta, GeorGIA 30322

E-mail address: skip@member.ams.org

$U R L:$ http://www.mathcs . emory.edu/ ${ }^{\text {skip/ }}$ 